\title{
The expression and significance of inflammatory cytokines and MMP-9 in the tears of the infected eye and contralateral uninfected eye in patients with fungal keratitis
}

\section{Shuang Zhang}

The First Affiliated Hospital of Nanchang University

\section{Hui-Min Wu}

The First Affiliated Hospital of Nanchang University

\section{Xiang-Ni Cao}

The First Affiliated Hospital of Nanchang University

\section{Xian-Qi Zhang}

Huazhong University of Science and Technology

Gui-ping Gao ( $\nabla$ ggp13732938636@163.com )

The First Affiliated Hospital of Nanchang University

\section{Research Article}

Keywords: fungal keratitis, cytokines, matrix metalloproteinase-9, inflammation, immunity

Posted Date: March 11th, 2021

DOI: https://doi.org/10.21203/rs.3.rs-269176/v1

License: (c) (1) This work is licensed under a Creative Commons Attribution 4.0 International License.

Read Full License 


\section{Abstract}

Background: We investigated bilateral tear cytokine levels including interleukin (IL)-13, IL-10, IL-17, tumor necrosis factor (TNF)- $\alpha$ and Matrix metalloproteinase-9 (MMP-9) in patients with fungal keratitis(FK). Meanwhile, we evaluated the relationship between the changes of tear cytokines with corneal perception and pain in infected eyes, and the relationship between tear cytokines and tear film function in contralateral uninfected eyes .

Methods : A total of 60(20 FK, 20 contralateral, 20 healthy controls) tear samples were collected prospectively and analyzed by enzyme linked immunosorbent assay(ELISA). Approximately 50 to 60 ul of tear samples in each case were collected. Meanwhile, we analyzed the changes of visual analogue scale(VAS), tear breakup time (TBUT), Schirmer I test (SIT) and corneal perception compared with healthy controls.

Results :The concentrations of IL-1 $\beta$, IL-10 and IL-17 increased in bilateral eyes compared with healthy controls $(P<0.05)$. The tear concentrations of MMP-9, TNF- $a$ only significantly increased in affected eyes $(\mathrm{P}<0.05)$. Patients with FK showed significant reduction in corneal perception of infected eyes compared with controls $(P<0.05)$. Corneal perception of the normal eyes in FK patients was slightly lower than that of control group, but there was not statistical difference $(P>0.05)$. TBUT and SIT of contralateral uninfected eyes were significantly lower than that of control group $(P<0.05)$, which were significantly correlated with levels of IL-1 $\beta, I L-17(P<0.05)$. SIT were also negatively correlated with MMP-9 $(P<0.05)$, while the levels of IL-1 $\beta$, IL-10, IL-17, TNF- $\alpha$ and MMP-9 in the tears of the healthy control group had no significant correlation with TBUT and SIT indicators $(P>0.05)$. The corneal perception and VAS score of the affected FK eyes showed correlation with IL-1 $\beta$, IL-17 and TNF- $\alpha(P<0.05)$.In addition, concentration of IL10 inversely was correlated with VAS $(P<0.05)$.

Conclusion: Proinflammatory tear cytokines are elevated in bilateral eyes with unilateral FK as associated with tear film function ,pain and corneal sensitivity.

\section{Background}

Fungal keratitis(FK) is one of the major vision-threatening disease, which affects public health worldwide, especially in developing countries[1]. The most common pathogens recognized in FK are Aspergillus spp., Fusarium spp.[2]. Most infections with these organisms are initiated in agricultural environment as a result of ocular surface trauma caused by plant material branches or insects contaminated with fungal spores[3] .These microorganisms will invade the cornea, destruct the protective physical barrier at the ocular surface and initiate an immune or inflammatory response. It has been suggested that immune regulation plays a vital role in the pathogenesis of FK[4]. The recruitment of inflammatory cells induced by microorganisms and inflammation will trigger the release of cytokines and chemokines. In the early stage of this disease, it may be related to the adhesion and invasion of mycotoxin, however ,the further damage may be connected with the strong immune inflammatory. A certain inflammatory response can 
remove fungi and promote the repair of tissue. However, long-term overreaction of the host immune response may magnify inflammation, release further attracts more immune cells and lead to corneal opacity, tissue damage, even perforation and vision loss $[5,6]$.Therefore, studying the inflammatory factors has a great significance to explain the pathogenesis of fungal keratitis, which provides help for FK patients to avoid devastating outcomes. There exists numerous of immunocompetent cells in corneal limbus and conjunctival tissues. Intraocular antigen and mycotoxins will contact the conjunctiva or corneal limbus, enter the systemic lymphatic system and activate the human immune system, which will cause immune inflammation in contralateral eye[7]. Although there are no reports about sympathetic ophthalmia happened in FK patients, some studies have shown related immune changes in contralateral eyes. The collection of intraocular samples such as aqueous humor is invasive, therefore we replaced them with tear samples to reflect the inflammatory response because it has little discomfort and is noninvasive[8]. Nowdays, tear samples are increasingly used to detect biomarkers of normal and diseased ocular surfaces, such as dry eye[9] and human microbial keratitis[10, 11]. In our study, We investigated bilateral tear cytokine levels and MMP-9 to evaluate immune response of bilateral eyes in patients with FK. Meanwhile, we evaluate the influence of these indicators on pain, corneal nerve and tear film changes.

\section{Methods}

\section{Participants}

There were a total of $20 \mathrm{FK}$ patients ( 12 males, 8 females) and 20 healthy controls ( 11 males, 9 females) who had already matched their sex, age, with FK patients in this study. All FK patients were diagnosed in the Ophthalmology Department of the hospital of a comprehensive university. Inclusion criteria of FK patients were in accordance with one of the following three items: (i) fungal hyphae and / or spores were observed under microscope after smeared by corneal scraping; (Fig.1); (ii) fungal hyphae or spores were found under corneal confocal microscope;(iii) the culture results of scraped specimens of fungal lesions were fungi.

Exclusion criteria for FK patients or healthy control subjects (i) systemic immunological or rheumatic diseases;(ii)diabetes;(iii) previously infected with viral or other keratitis;(iv) a history of eye trauma and/or surgery;(v) other ocular surface diseases affecting the secretion of tear cytokines, such as Pterygium or conjunctivitis;(vi) with dry eye symptoms in the past.

This study was approved by the ethics committee of a comprehensive university hospital. All the methods were performed in line with the Declaration of Helsinki. In addition, every patient involved in this study had been informed of the whole study design and signed the informed consent.

\section{Tear collection}

A total of 60 (20 FK, 20 contralateral, 20 healthy controls) tear samples were collected .As for healthy controls, the tear samples were always selected from right eyes. Tear samples were collected in the same 
time frame(between 7.00 and $8.00 \mathrm{am}$ ) without stimulation .By using $10 \mathrm{ul}$ glass capillary micropipettes(Drummond, USA), approximately 50 to 60 ul of tear samples in each case was collected. Every time we used a new micropipette sterile tip for each eye. Reserved 50 to 60 ul tear samples were transferred into sterile microfuge tubes and stored at $-20{ }^{\circ} \mathrm{C}$ until assaying for the cytokine.

\section{Enzyme -linked immunosorbent assay(ELISA)}

IL-1 $\beta$, IL-6, TNF- $a$, IL-10 and MMP-9 kits (R\&D systems, Minneapolis, USA『were used to detect the expression levels of IL-1 $\beta, \mathrm{IL}-10, \mathrm{IL}-17$, TNF- $a$ and MMP-9 in tears. The operation was according to the instructions of ELISA kit, as follows: (1)Add standard: Set Standard wells, testing sample wells. Add standard $50 \mu \mathrm{l}$ to standard well.(2)Add Sample: Add testing sample $10 \mu \mathrm{l}$ then add $40 \mu \mathrm{l}$ of Sample Diluent to testing sample well; Blank well doesn't add anything. (3) Add 100 $\mu$ l of HRP-conjugate reagent to each well, cover with an adhesive strip and incubate for 60 minutes at $37^{\circ} \mathrm{C}$. (4) Aspirate each well and wash, repeating the process four times for a total of five washes. Wash by filling each well with Wash Solution $(400 \mu \mathrm{l})$ using a squirt bottle, manifold dispenser or auto washer. Complete removal of liquid at each step is essential to good performance. After the last wash, remove any remaining Wash Solution by aspirating or decanting. Invert the plate and blot it against clean paper towels. (5) Add chromogen solution A 50 $\mu$ l

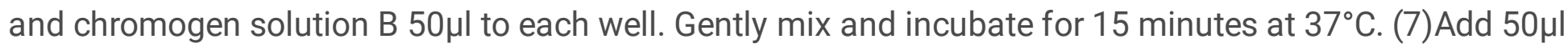
Stop Solution to each well. The color in the wells should change from blue to yellow. (8)Read the Optical Density (O.D.) at $450 \mathrm{~nm}$ using a microtiter plate reader within 15 minutes.

\section{Visual analogue scale (VAS)}

The pain degree of the patients was measured by VAS developed by the National Institutes of Health[12]. The degree of pain on a scale of 0-10: 0 point: no pain; Less than 3 point: mild pain but bearable; 4-6 point: tolerate pain though affecting sleep; 7-10 point: unbearable pain ,which affects appetite and sleep.

\section{Tear film examination}

The examination of the liquid was mainly carried out from two items: tear breakup time(TBUT) and Schirmer I test(SIT).(1)TBUT: TBUT reflects the stability of tear film. Fluorescein sodium strips(Tianjin Jingming New Technological Development Co., Ltd.) was placed in the lower eyelid conjunctival sac for about 1-2 seconds, After taking out the test paper, we told patient to blink several times, then look straightly ahead and try to prolong opening time of the eye. We observed the tear film under slit lamp cobalt blue light, and calculated time from the last blink after natural opening eyes until appearing the first dry spot on the corneal surface. After repeated measurement for 3 times in each eye, the average value was taken to record the results.(2)SIT: Schirmer I test reflected the basal secretion of tears. We folded the Schirmer tear test strips of $5 \mathrm{~mm} \times 30 \mathrm{~mm}$ standard(Tianjin Jingming New Technological Development Co., Ltd.) from the opening of the notch for $5 \mathrm{~mm}$. Then we placed it at the junction of the conjunctival sac of the lower eyelid (gently, minimize stimulation), and told the patient to look down and close his eyes gently. 5 minutes later, we took out the filter strip and recorded the soaking length. 


\section{Corneal perception examination}

The Coehet-Bonnet keratometer ( Luneau SA company ,France) was used to detect the corneal sensitivity ,of which the diameter of the fiber was $0.12 \mathrm{~mm}$, and the longest length was $60 \mathrm{~mm}$. After telling the patient to sit upright, and look straight forward, we contacted the center of the cornea vertically with the fiber endings. The examiner saw the fiber bending with the naked eye. The positive feedback was that the patient felt the sensation of foreign body in the cornea. Starting from $60 \mathrm{~mm}$, the length was reduced by 5 $\mathrm{mm}$ each time until the patient showed a positive reaction, and the average value was taken 3 times. All examinations were performed by the same physician.

\section{Statistical analysis}

Statistical analyses were performed by SPSS software(version 22, SPSS Inc., Chicago, Illinois, USA). The basic information (age, weight) between the patient and the healthy control group was compared by independent sample t-test, while gender differences were by Fisher's accurate test .The differences among the three groups were compared by Kruskal-Wallis $\mathrm{H}$ test, further comparison between groups using Nemenyi test, and the differences of BUT between the two groups were compared by Mann-Whitner U test. We used prism for Windows version 7(GraphPad Software, Inc., San Diego, CA, USA) to draw statistical figures. Spearman's correlation analysis was used to evaluate the correlation between VAS, TBUT , SIT, corneal Perception and cytokine level. For each test, differences were considered statistically significant when $\mathrm{P}<0.05$ and data were presented as mean \pm SD.

\section{Results}

\section{Participants}

There were no marked differences in weight $(P=0.677)$, age $(P=0.982)$ and $\operatorname{sex}(P=1.000)$ between the FK group and healthy group. More details are showen in Table 1.Clinical data of FK patients are presented in Table 2. We collected 20 fungal keratitis patients, we found the most frequent causative microorganism of fungal keratitis was Fussarium spp.; The duration of the illness ranged from 3 to 60 days. The most common cause was damage from plants or insects contaminated by fungal spores. Fig. 1 shows the slit-lamp photographs of infected eye and contralateral uninfected eye from the FK patient.

Table1 Demographics and clinical measurements of FK and HC groups

\begin{tabular}{llcll}
\hline & FK & HCs & $t$ & $P^{*}$ \\
\hline Male/Female & $12 / 8$ & $11 / 9$ & N/A & $1.000 \#$ \\
age $Y$ Year $\square$ & $53.65 \pm 14.64$ & $52.98 \pm 14.29$ & 0.273 & $0.982^{*}$ \\
weight $₫ \mathrm{Kg} \square$ & $60.25 \pm 6.43$ & $61.05 \pm 7.08$ & -0.374 & $0.677^{*}$ \\
\hline
\end{tabular}

Notes:N/A means data is not available, * represents independent sample t-test, \# represents Fisher's exact test.When $P>0.05$, the difference is not significant. 
Table 2 Demographic, clinical aspects in fungal keratitis patients

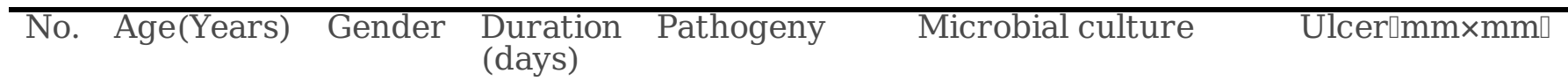

\begin{tabular}{|c|c|c|c|c|c|c|}
\hline 1 & 76 & Female & 3 & None & Fusarium & $5 \times 6$ \\
\hline 2 & 70 & Male & 15 & Foreign body & Fusarium & $6 \times 7$ \\
\hline 3 & 56 & Male & 15 & Plant injury & Colletotrichum & $3 \times 4$ \\
\hline 4 & 25 & Male & 7 & Insect trauma & Aspergillus fumigatus & $3 \times 3$ \\
\hline 5 & 42 & Female & 30 & None & Fusarium & $4 \times 5$ \\
\hline 6 & 64 & Male & 14 & Plant injury & Fungal hyphae & $3 \times 4$ \\
\hline 7 & 54 & Female & 30 & Plant injury & Fungal hyphae & $3 \times 2$ \\
\hline 8 & 47 & Female & 12 & Plant injury & Fusarium & $2 \times 2$ \\
\hline 9 & 68 & Female & 14 & Plant injury & Curvularia lunata & $6 \times 4$ \\
\hline 10 & 61 & Male & 60 & Plant injury & Fusarium & $4 \times 4$ \\
\hline 11 & 52 & Male & 7 & None & Fusarium & $5 \times 4$ \\
\hline 12 & 44 & Male & 30 & Foreign body & Aspergillus fumigatus & $4 \times 4$ \\
\hline 13 & 26 & Female & 5 & None & Fusarium & $2 \times 4$ \\
\hline 14 & 56 & Male & 2 & None & Fusarium & $4 \times 5$ \\
\hline 15 & 67 & Female & 7 & Plant injury & Aspergillus fumigatus & $3 \times 3$ \\
\hline 16 & 32 & Male & 3 & Plant injuru & Fusarium & $4 \times 3$ \\
\hline 17 & 58 & Male & 15 & Plant injury & Curvularia lunata & $2 \times 3$ \\
\hline 18 & 71 & Female & 5 & None & Fungal hyphae & $5 \times 4$ \\
\hline 19 & 59 & Male & 15 & Plant injurury & Fungal hyphae & $2 \times 2$ \\
\hline 20 & 45 & Male & 28 & Plant injury & Fusarium & $3 \times 3$ \\
\hline
\end{tabular}

\section{Tear Cytokine Concentrations}

Compared with healthy controls, the concentrations of IL-1 $\beta, I L-10$ and IL-17 in tears from FK patients increased in bilateral eyes $(\mathrm{P}<0.05)$, and the levels of IL-10 in tears of the contralateral uninfected eye were higher than those in infected eyes, the concentrations of TNF- $a$ and MMP-9 only increased in affected FK eyes $(P<0.05)$, but not contralateral eyes $(P>0.05)$. The detailed tear cytokine concentrations measured by ELISA are shown in Fig. 2 and Table 3.

Table 3 Cytokine levels in tears from fungal keratitis patients and controls₫ ${ }^{\mathrm{X}} \pm \mathrm{SD} \square$ 


\begin{tabular}{|c|c|c|c|c|c|c|c|c|c|}
\hline & Group A & Group B & Group C & $\mathrm{A}$ vs B & & A vs C & $\overline{\mathrm{B}}$ & $\overline{S C}$ & \\
\hline & & & & C2 & $\mathrm{P}$ & c2 & $\mathrm{P}$ & c2 & $\bar{P}$ \\
\hline $\mathrm{IL}-\beta \square \mathrm{pg} / \mathrm{ml} \square$ & $72.65 \pm 13.44$ & $23.38 \pm 5.14$ & $9.62 \pm 3.66$ & $\begin{array}{l}13.59 \\
<0.01\end{array}$ & & 53.74 & $<0.01$ & 13.28 & $<0.01$ \\
\hline IL-10 $\square \mathrm{pg} / \mathrm{ml} \square$ & $32.63 \pm 5.53$ & $42.88 \pm 6.34$ & $14.70 \pm 3.72$ & 7.29 & $<0.05$ & 16.45 & $<0.01$ & 45.62 & $<0.01$ \\
\hline IL-17ロpg/ml & $39.74 \pm 13.44$ & $20.92 \pm 4.65$ & $12.83 \pm 5.10$ & $\begin{array}{l}10.52 \\
<0.05\end{array}$ & & 39.50 & $<0.01$ & 9.25 & 0.01 \\
\hline $\begin{array}{l}\text { TNF- } \alpha \\
\square \mathrm{pg} / \mathrm{ml} \square\end{array}$ & $54.34 \pm 15.33$ & $19.58 \pm 8.90$ & $14.17 \pm 6.09$ & $\begin{array}{l}17.07 \\
<0.01\end{array}$ & & 41.00 & $<0.01$ & 5.16 & $>0.05$ \\
\hline $\begin{array}{l}\text { MMP-9 } \\
\square \mathrm{ng} / \mathrm{ml} \square\end{array}$ & $10.41 \pm 1.82$ & $4.53 \pm 1.37$ & $4.37 \pm 1.50$ & $\begin{array}{l}28.56 \\
<0.01\end{array}$ & & 30.43 & $<0.01$ & 0.03 & $>0.05$ \\
\hline
\end{tabular}

Notes: Group A represents the infected eye group, group B represents the contralateral uninfected eye group, and group C represents the healthy control group; The differences among the three groups were compared by Kruskal-Wallis $\mathrm{H}$ test, further comparison between groups using Nemenyi test, differences were considered statistically significant at $\mathrm{P}<0.05$.

Abbreviations : IL, Interleukin;MMP-9, Matrix metalloproteinase-9; TNF, Tumor necrosis factor.

\section{Corneal perception and tear film function}

The corneal perception in affected FK eye was significantly lower than that of the healthy controls, and the corneal perception of the contralateral uninfected eye was slightly lower than that of the control group, but there was not statistical difference $(P>0.05)$, The TBUT and Schirmer strip of the contralateral uninfected eye were significantly lower than that of the healthy control group, and the difference was statistically significant $(P<0.05)$. The Schirmer strip of the affected FK eyes were significantly longer than healthy controls $(P<0.05)$. More details are shown in Fig.3 ,Table 4.

Table 4 Cytokine levels in tears from fungal keratitis patients and controls ${ }^{`} \mathrm{X} \pm \mathrm{SD} \square$ 


\begin{tabular}{|c|c|c|c|c|c|c|c|c|c|}
\hline & Group A & Group B & Group C & $\mathrm{A}$ vs B & & A vs C & $\overline{\mathrm{B}}$ & $\overline{S C}$ & \\
\hline & & & & C2 & $\mathrm{P}$ & c2 & $\mathrm{P}$ & c2 & $\bar{P}$ \\
\hline $\mathrm{IL}-\beta \square \mathrm{pg} / \mathrm{ml} \square$ & $72.65 \pm 13.44$ & $23.38 \pm 5.14$ & $9.62 \pm 3.66$ & $\begin{array}{l}13.59 \\
<0.01\end{array}$ & & 53.74 & $<0.01$ & 13.28 & $<0.01$ \\
\hline IL-10 $\square \mathrm{pg} / \mathrm{ml} \square$ & $32.63 \pm 5.53$ & $42.88 \pm 6.34$ & $14.70 \pm 3.72$ & 7.29 & $<0.05$ & 16.45 & $<0.01$ & 45.62 & $<0.01$ \\
\hline IL-17ロpg/ml & $39.74 \pm 13.44$ & $20.92 \pm 4.65$ & $12.83 \pm 5.10$ & $\begin{array}{l}10.52 \\
<0.05\end{array}$ & & 39.50 & $<0.01$ & 9.25 & 0.01 \\
\hline $\begin{array}{l}\text { TNF- } \alpha \\
\square \mathrm{pg} / \mathrm{ml} \square\end{array}$ & $54.34 \pm 15.33$ & $19.58 \pm 8.90$ & $14.17 \pm 6.09$ & $\begin{array}{l}17.07 \\
<0.01\end{array}$ & & 41.00 & $<0.01$ & 5.16 & $>0.05$ \\
\hline $\begin{array}{l}\text { MMP-9 } \\
\square \mathrm{ng} / \mathrm{ml} \square\end{array}$ & $10.41 \pm 1.82$ & $4.53 \pm 1.37$ & $4.37 \pm 1.50$ & $\begin{array}{l}28.56 \\
<0.01\end{array}$ & & 30.43 & $<0.01$ & 0.03 & $>0.05$ \\
\hline
\end{tabular}

Notes: Group A represents the infected eye group, group B represents the contralateral uninfected eye group, and group C represents the healthy control group; The differences among the three groups were compared by Kruskal-Wallis $\mathrm{H}$ test, further comparison between groups using Nemenyi test, differences were considered statistically significant at $\mathrm{P}<0.05$.

Abbreviations : IL, Interleukin;MMP-9, Matrix metalloproteinase-9; TNF, Tumor necrosis factor;.

\section{Correlation analysis}

\section{Correlation of inflammatory cytokines in tears with corneal perception and VAS}

Tear concentrations of IL-1 $\beta$, IL-17 and TNF- a were positively correlated with VAS $(R=0.873, P<0.001$; $R=0.748, P<0.001, R=0.809, P<0.001$, respectively;Fig.4A-B.D). While IL-10 was correlated inversely with $\operatorname{VAS}(R=-0.668, P=0.001, F i g .4 C)$, tear concentrations of IL-1 $\beta, I L-17$ and TNF- a were correlated inversely with corneal perception $(R=-0.492, P<0.001 ; R=-0.686, P=0.001 ; R=-0.598, P<0.05$, respectively; Fig.4EF.H).IL-10 was not correlated with corneal perception significantly ( $P>0.05$, Fig.4G). Tear concentrations of MMP-9 was also not correlated with VAS and corneal perception significantly $(P>0.05)$.

\section{Correlation between inflammatory cytokines and tear film function in the contralateral uninfected tear}

The levels of IL-1 $\beta, I L-17$ and MMP-9 were inversely correlated with Schirmer strip( $R=-0.822, P<0.001$, $R=-0.811, P<0.001, R=-0.508, P<0.05$, respectively; Fig.5A-C). The tear concentrations of IL-1 $\beta$, IL-17 were also inversely correlated with TBUT $(R=-0.619, P<0.05 ; R=-0.551$, respectively; Fig.5D-F). MMP-9 was did not correlated with TBUT significantly $(P=0.058$, Fig.5F). There were no significant correlations between other cytokines, and Schirmer strip and ,TBUT $(P>0.05)$. 


\section{Correlation between tear inflammatory cytokines and tear film function in healthy controls}

There was no significant correlation between tear film function and the levels of IL-1 $\beta$, IL-10, IL-17, TNF- $a$ and MMP-9 in normal control group (fig. 6, Fig. 7) $(r=-0.323-0.134, P>0.05)$.

\section{Discussion}

When fungi invades the body, it will trigger a series of inflammatory events, including infiltration of inflammatory cells and factors. IL-1 $\beta$ is produced by immune cells and mucosal epithelial cells of the ocular surface, it is one of the crucial proinflammatory and inflammation mediator[13] involving in fungal-induced corneal injury[11]. TNF-a is a crucial proinflammatory mediator in a variety of corneal diseases, TNF-a can disrupt the barrier function of human corneal epithelial cells and promote ocular inflammation [14]. MMP-9, known as gelatinase $B$. is distributed in corneal stroma as associated with epithelial repair and remodeling in physiological environment. Under pathological conditions, it is actively secreted by neutrophils in the process of injury and inflammation, and excessive MMP-9 is expressed in corneal epithelial cells, which will cause severe tissue damage [15].IL-10 is a key negative regulator of inflammation and has immunosuppressive activity[16]. It can regulate innate and adaptive immune cells and prevent the pathological development of immunity in different ways[17]. In the fungal keratitis model, the expression of IL-10 in the cornea increased at first and then decreased after a period of time [18]. IL17 is secreted by Thl7 cells, neutrophils and glial cells can also secrete a few [19]. A large amount of IL-17 expression was found in human cornea with filamentous fungal keratitis[20]. IL-17 can alleviate the severity of fungal keratitis by the expression of CX43 in corneal peripheral vascular endothelial cells[21]. Therefore, it suggests that low levels of IL-10, IL-17 was useful to enhance the corneal defense system which is to protect against disease onset, whereas such an episode, a high level of IL-10,IL-17 assists in dampening the inflammatory response, and minimizing tissue destruction and scarring[22].Therefore, the increase of anti-inflammatory factors represents the ability to buffer inflammatory response and limit collateral damage to the host [23].

In our study, IL-1 $\beta$, IL-6 and TNF- $a$ showed a positive correlation with VAS, while IL-10 showed a negative correlation with VAS. IL-1 $\beta$ had been shown to induce additional proinflammatory mediators, such as IL-6, NO, prostaglandin E2(PGE-2), and cyclooxgenase-2(COX-2)[24]. COX-2 and PGE-2 are crucial pain factors. Meanwhile,IL-1 $\beta$ can modulate neuronal intracellular calcium signaling and activates astrocytes, which can further enhance and prolong neuroinflammation-induced chronic pain[25]. IL-17 can promote astrocyte proliferation and proinflammatory cytokines secretion, which are related to the neuropathic pain[26]. TNF- $a$ is a crucial regulator of inflammatory response, which participates in the production of pain[27]. As an anti-inflammatory factor, IL-10 plays an essential role in neuropathic pain, which can reduce the pain response by inhibiting the release of pro-inflammatory factors[28]. This is consistent with our study that pain scores decrease with the increase of anti-inflammatory factors. In addition, a new study shows that pain behavior can be reduced by injecting recombinant IL-10 into the trigeminal ganglion[29], which provides ideas for trigeminal neuralgia of keratitis patients in future. Besides, these inflammatory cytokines are inversely proportional to corneal perception, it may be related to mycotoxin 
and inflammatory cytokine-mediated damage, and may lead to the damage of trigeminal nerve. However, pain sensitivity may decrease with post infection corneal ulcer aggravation, which may be caused by decreasing corneal nerve density in FK patients[30].

The expression of a large number of inflammatory cytokines can directly damage the corneal barrier and lead to changes in tear film function. Moreover, the interaction between dry eye and inflammation will further lead to the activation of ocular surface cascade immune inflammation [31, 32]. These may influence the tear film function of contralateral uninfected eyes. Study have showed that there are relations between tear inflammatory cytokines and dry eye [33]. The increase of IL-10 was also found in tear and conjunctival with dry eye patients [34]. TFOS DED II guilds showed IL-1 $\beta$, IL-17, TNF- $\alpha$ and MMPs played a vital role in the pathogenesis of dry eye[35]. IL-17 may be involved in the immune pathogenesis of xerophthalmia and destroy the corneal epithelial barrier to promote the occurrence of dry eye. Meanwhile, drying stress can induce Th1 inflammatory cytokines on the ocular surface and destroy the corneal epithelial barrier associated with Th17[36]. This is consistent with our research. This indicates further that the increase of related inflammatory factors in the contralateral eyes will cause corresponding changes in tear film function. Due to stimulation such as injury, toxin and immunity, which may stimulate the lacrimal gland nerves, the tear secretion of the affected eyes will be increased reflexively. Therefore, We did not measure TBUT of the affected eye.

Study has shown that when unilateral eye is infected or damaged, the nerve growth factor[37], inflammatory cytokines[10], dendritic cell[38], the nerve density began to decrease [39] and leukocyte exudation phenomenon can be detected in the contralateral uninfected eyes [40], which further indicates that there is a connection in bilateral eye, and the corresponding immune changes will also occur in the contralateral uninfected eye. In the tear cytokine study of keratitis [10], it was found that the expression level of IL-10,IL-17 in the contralateral eye increased; The increase of IL-10,IL-17 may be a preventive mechanism to avoid infection in the contralateral eye. Furthermore, In the study of cataract surgery, IL-1 $\beta$ was elevated in contralateral eye, which may be associated with pain[41]. This manifests that pain and immunity are also interrelated. In the herpes simplex virus study, it was observed that when monocular infectious keratitis occurs, the tear film indexes such as SIT, TBUT will decrease, the tear osmotic pressure increased, and the nerve density will decrease[42], which is consistent with our study. The peripheral nervous system can regulate the activation, deployment and homeostasis of the immune system, and initiate adaptive immunity, which indicates that there is also a connection between the corneal nerve and immune system[43]. Patients with depression has an increase in tear inflammatory cytokines and shows dry eye symptoms [9]. As we all know, low sleep quality and discomfort of eye caused by vision loss, pain makes the FK patients in a state of anxiety, which increases the probability to develop dry eye syndrome in contralateral uninfected eyes. Although the patient does not have obvious subjective feelings, this may be covered by pain of infected eye.

\section{Conclusion}


Our study demonstrated that unilateral FK can result in bilateral inflammatory tear inflammatory cytokines alterations, which indicates that the immune response links between bilateral eyes. Meanwhile, these inflammatory factors appear to be related to pain and corneal perception, which indicates that immune-nerve-pain is interactive and interrelated. The long-term uncured fungal infection will potentially influence the contralateral uninfected ocular surface environment in patients with unilateral FK. They are more likely to suffer from dry eye than healthy people in future. This also indicates researchers should be careful to use the contralateral uninfected eyes as a healthy control.

\section{Declarations}

\section{Ethics approval and consent to participate}

The experimental protocol was established, according to the ethical guidelines of the Helsinki Declaration and was approved by the Human Ethics Committee of The First Affiliated Hospital of Nanchang University. Written informed consent was obtained from individual or guardian participants.

\section{Consent for publication}

All data generated or analyzed during this study from patients are included in this published article.

Every patient involved in this study had been informed of the whole study design and signed the informed consent. Patients were consent to publish these pictures.

\section{Availability of data and materials}

The datasets used or analysed during the current study are available from the corresponding author on reasonable request.

\section{Competing interests}

This was not an industry supported study. The authors report no conflicts of interest in this work.

\section{Funding}

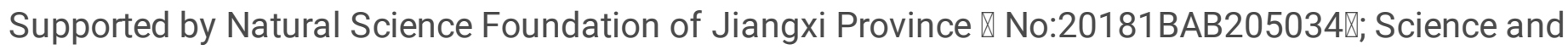
Technology Design of Jiangxi Education Department Projects $₫$ No: GJJ170048区.

\section{Authors' contributions}

Shuang Zhang and Gui-Ping Gao analyzed and interpreted the patient data regarding the hematological disease and the transplant. Shuang Zhang, Hui-Min Wu and Xiang-Ni Cao performed the histological examination of the kidney, and Shuang Zhang was a major contributor in writing the manuscript. Xian-Qi Zhang was responsible for polishing the article.All authors read and approved the final manuscript.

\section{Acknowledgements}


We appreciated Yi Shao for guidance of the manuscript.

\section{References}

1. Xie L, Zhong W, Shi W, Sun S: Spectrum of Fungal Keratitis in North China. Ophthalmology 2006, 113(11):1943-1948.

2. Chirinossaldaña P: Infectious keratitis: microbiological and antibiotic sensitivity trends. Second Annual Report of the Ocular Microbiology Study Group from the Instituto de Oftalmología "Conde de Valenciana". Revista Mexicana De Oftalmologia 2013, 87(2):100-109.

3. Fodor M, Facsk, oacute, Andrea, Rajnav, ouml, Lgyi, Eacute, va, aacute et al: Enhanced release of IL-6 and IL-8 into tears in various anterior segment eye diseases. Ophthalmic Research 2006, 38(4):182188.

4. Chang DC, Grant GB, O,Donnell K, Wannemuehler KA, Noble-Wang J, Rao CY, Jacobson LM, Crowell CS, Sneed RS, Lewil FMT et al: Multistate outbreak of Fusarium keratitis associated with use of a contact lens solution. American Journal of Ophthalmology 2006.

5. Wu M, Peng A, Sun M, Deng Q, Huang X: TREM-1 Amplifies Corneal Inflammation after Pseudomonas aeruginosa Infection by Modulating Toll-Like Receptor Signaling and Th1/Th2-Type Immune Responses. 2011.

6. Underhill D, Pearlman E: Immune Interactions with Pathogenic and Commensal Fungi: A Two-Way Street. Immunity 2015, 43(5):845-858.

7. Rua D, Pohlmann D, Pleyer U: Sympathetic Ophthalmia - a Contribution to Immunology, Clinic and Current Imaging. Klinische Monatsblatter fur Augenheilkunde 2020, 237(9):1060-1069.

8. Zhang Y, Liang Q, Liu Y, Pan Z, Lu Q: Expression of cytokines in aqueous humor from fungal keratitis patients. Bmc Ophthalmology 2018, 18(1):105.

9. Małgorzata, Mrugacz, Lucyna, Ostrowska, Anna, Bryl, Agata, Szulc, Beata, Zelazowska-Rutkowska: Pro-inflammatory cytokines associated with clinical severity of dry eye disease of patients with depression. Advances in Medical Sciences 2017.

10. Yamaguchi T, Calvacanti BM, Cruzat A, Qazi Y, Ishikawa S, Osuka A, Lederer J, Hamrah P: Correlation between human tear cytokine levels and cellular corneal changes in patients with bacterial keratitis by in vivo confocal microscopy. Invest Ophthalmol Vis Sci 2014, 55(11):7457-7466.

11. Santacruz C, Linares M, Garfias Y, Loustaunau L, Pavon L, Perez-Tapia S, Jimenez-Martinez M: Expression of IL-8, IL- 6 and IL-1 $\beta$ in Tears as a Main Characteristic of the Immune Response in Human Microbial Keratitis. International Journal of Molecular Sciences 2015, 16(3):4850-4864.

12. Gulia A, Dhamija E, Kumar M, Thulkar S, Bhatnagar S: Impact of Early Intervention in Pain Management in Cancer Patients- A Randomised Controlled Study in a Tertiary Care Cancer Hospital. The Clinical journal of pain 2021.

13. Gao X, Zhao G, Li C, Lin J, Jiang N, Wang Q, Hu L, Xu Q, Peng X, He K: LOX-1 and TLR4 affect each other and regulate the generation of ROS in A. fumigatus keratitis. International 
Immunopharmacology 2016.

14. Kimura K: [Molecular mechanism of the disruption of barrier function in cultured human corneal epithelial cells induced by tumor necrosis factor-alpha, a proinflammatory cytokine]. Nihon Ganka Gakkai Zasshi 2010, 114(11):935-943.

15. Immunohistochemical study of matrix metalloproteinases-2 and $-\mathbf{9}$, macrophage inflammatory protein-2 and tissue inhibitors of matrix metalloproteinases- 1 and -2 in normal, purulonecrotic and fungal infected equine corneas. Veterinary Ophthalmology 2010, 13(2):81-90.

16. Ghasemi H, Ghazanfari T, Yaraee R, Owlia P, Hassan ZM, Faghihzadeh S: Roles of IL-10 in ocular inflammations: a review. Ocular Immunology \& Inflammation 2012, 20(6):406-418.

17. Wei H, Li B, Sun A, Guo F: Interleukin-10 Family Cytokines Immunobiology and Structure. Advances in experimental medicine and biology 2019, 1172:79-96.

18. Na, Li, Cheng-Ye, Che, Li-Ting, Hu, Jing, Lin, Qing, Wang: Effects of COX-2 inhibitor NS-398 on IL-10 expression in rat fungal keratitis. International Journal of Ophthalmology(English Edition) 2011, 02(v.4):55-59.

19. Sivaganesa KR, Chairut V, Venkatesh PN, Kuppamuthu D, Eric P, Prajna L: Interleukin 17 Expression in Peripheral Blood Neutrophils From Fungal Keratitis Patients and Healthy Cohorts in Southern India. Journal of Infectious Diseases 2015(1):130-134.

20. Me R, Gao N, Dai C, Yu F: Pseudomonas aeruginosalL-17 Promotes Keratitis in C57BL/6 Mouse Corneas. Journal of immunology (Baltimore, Md: 1950) 2020, 204(1):169-179.

21. Qin XH, Ma X, Fang SF, Zhang ZZ, Lu JM: IL-17 produced by Th17 cells alleviates the severity of fungal keratitis by suppressing CX43 expression in corneal peripheral vascular endothelial cells. Cell Cycle 2019:1-14.

22. Carnt N, Cipriani V, Stapleton F, Calder V, Willcox M: Association study of single nucleotide polymorphisms in IL-10 and IL-17 genes with the severity of microbial keratitis. Contact lens \& anterior eye: the journal of the British Contact Lens Association 2019, 42(6):658-661.

23. Asadullah K, Sterry W, Volk H: Interleukin-10 therapy-review of a new approach. Pharmacological reviews 2003, 55(2):241-269.

24. Lee Y-A, Choi HM, Lee S-H, Yang H-I, Yoo MC, Hong S-J, Kim KS: Synergy between adiponectin and interleukin-1 $\beta$ on the expression of interleukin-6, interleukin-8, and cyclooxygenase- 2 in fibroblast-like synoviocytes. Experimental \& Molecular Medicine 2012, 44(7):440-447.

25. Gajtkó A, Bakk E, Hegedús K, Ducza L, Holló K: IL-1 $\beta$ Induced Cytokine Expression by Spinal Astrocytes Can Play a Role in the Maintenance of Chronic Inflammatory Pain. Frontiers in physiology 2020, 11:543331.

26. Sun C, Zhang J, Chen L, Liu T, Xu G, Li C, Yuan W, Xu H, Su Z: IL-17 contributed to the neuropathic pain following peripheral nerve injury by promoting astrocyte proliferation and secretion of proinflammatory cytokines. Molecular medicine reports 2017, 15(1):89-96.

27. Xu Q, Yaksh TL: A brief comparison of the pathophysiology of inflammatory versus neuropathic pain. Current Opinion in Anaesthesiology 2011, 24(4):400-407. 
28. Ellis A, Bennett DLH: Neuroinflammation and the generation of neuropathic pain. British Journal of Anaesthesia (1):26-37.

29. Iwasa T, Afroz S, Inoue M, Arakaki R, Oshima M, Raju R, Waskitho A, Inoue M, Baba O, Matsuka Y: IL10 and CXCL2 in trigeminal ganglia in neuropathic pain. Neuroscience letters 2019, 703:132-138.

30. Ren CJ, Zhou YF, Wu Y, Peng XD, Li C, Wang Q, Zhu GQ, You J, Zhang J, Zhao GQ: Changes in corneal

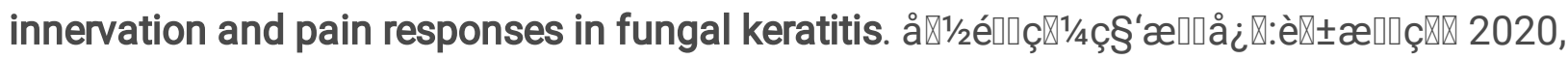
013(001):P.1-6.

31. Xu N, Cui Y, Fu D, Sun F: Tear inflammatory cytokines and ocular surface changes in patients with active thyroid eye disease treated with high-dose intravenous glucocorticoids. Journal of Endocrinological Investigation 2020(5-6).

32. Roda M, Corazza I, Reggiani MLB, Pellegrini M, Versura P: Dry Eye Disease and Tear Cytokine Levels -A Meta-Analysis. International Journal of Molecular Ences 2020, 21(9):3111.

33. VanDerMeid K, Su S, Ward K, Zhang J: Correlation of tear inflammatory cytokines and matrix metalloproteinases with four dry eye diagnostic tests. Investigative ophthalmology \& visual science 2012, 53(3):1512-1518.

34. Pflugfelder SC, Stern ME: Mucosal environmental sensors in the pathogenesis of dry eye. Expert Rev Clin Immunol 2014, 10(9):1137-1140.

35. Bron AJ, Paiva CSD, Chauhan SK, Bonini S, Sullivan DA: TFOS DEWS II pathophysiology report. Ocular Surface 2017, 15(3):438.

36. Sadrai Z, Stevenson W, Okanobo A, Chen Y, Dohlman TH, Hua J, Amparo F, Chauhan SK, Dana R: PDE4 inhibition suppresses IL-17-associated immunity in dry eye disease. Investigative Ophthalmology \& Visual Science 2012, 53(7):3584.

37. Paunicka KJ, Mellon J, Robertson D, Petroll M, Brown JR, Niederkorn JY: Severing Corneal Nerves in One Eye Induces Sympathetic Loss of Immune Privilege and Promotes Rejection of Future Corneal Allografts Placed in Either Eye. American Journal of Transplantation Official Journal of the American Society of Transplantation \& the American Society of Transplant Surgeons 2015, 15(6):1490-1501.

38. Hendricks RL: Corneal Nerve Damage In Microbial Keratitis. Investigative Ophthalmology \& Visual Science 2015, 56(11):6621.

39. Cruzat A, Schrems WA, Schrems-Hoesl LM, Cavalcanti BM, Baniasadi N, Witkin D, Pavan-Langston D, Dana R, Hamrah P: Contralateral Clinically Unaffected Eyes of Patients With Unilateral Infectious Keratitis Demonstrate a Sympathetic Immune Response. Investigative Ophthalmology \& Visual Science 2015, 56(11).

40. Vasanthi M, Prajna NV, Lalitha P, Mahadevan K, Muthukkaruppan V: A pilot study on the infiltrating cells and cytokine levels in the tear of fungal keratitis patients. Indian Journal of Ophthalmology 2007, 55(1):27.

41. Zhu X, Wolff D, Zhang K, He W, Sun X, Lu Y, Zhou P: Molecular Inflammation in the Contralateral Eye After Cataract Surgery in the First Eye. Investigative ophthalmology \& visual science 2015, 56(9):5566-5573. 
42. Ma X, Lu Y: Bilateral tear film alterations in patients with unilateral quiescent herpes simplex keratitis. Acta ophthalmologica 2017.

43. Ordovas-Montanes J, Rakoff-Nahoum S, Huang S, Riol-Blanco L, Barreiro O, Andrian UHV: The Regulation of Immunological Processes by Peripheral Neurons in Homeostasis and Disease. Other 2015, 36(10).

\section{Figures}
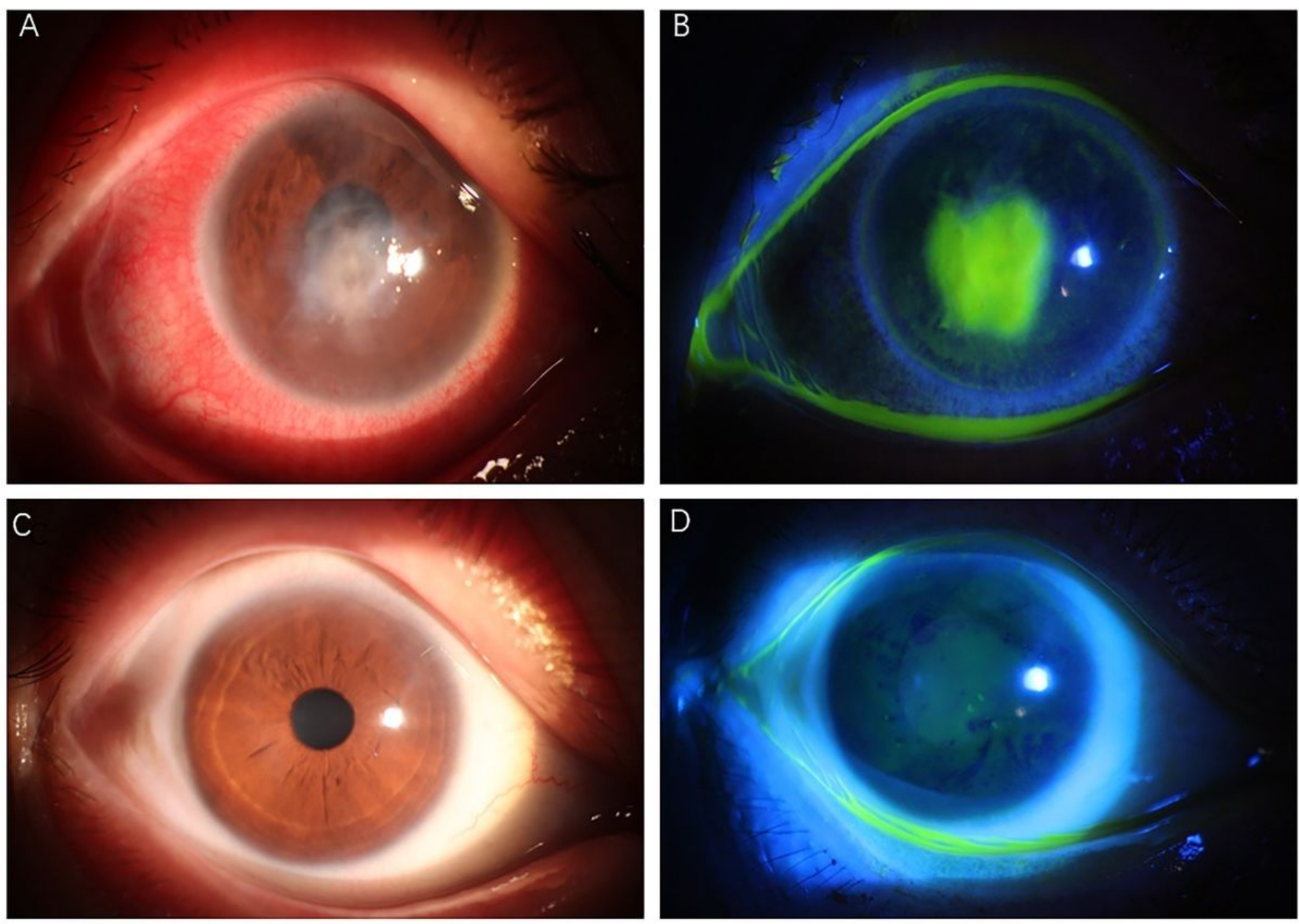

\section{Figure 1}

Slit lamp images from the fungal keratitis patient Figure 1A: We can see toothpaste-like appearance, pseudopodia and immune ring and other typical fungal infections symptoms .Figure 1B: The image of the infected eye after fluorescent staining, which shows a large of stained cornea area. Figure 1C: The image of contralateral uninfected eye without any obvious abnormality. While Figure 1D shows that cornea is slightly stained after fluorescent staining, and tear film is instability. 
A

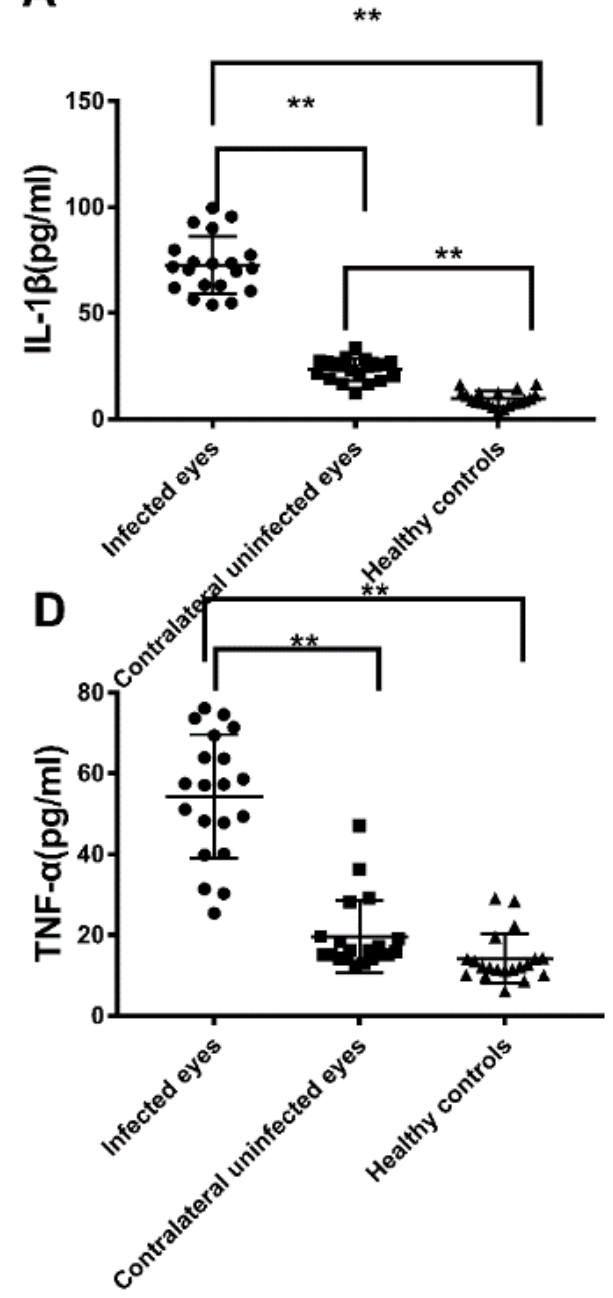

B

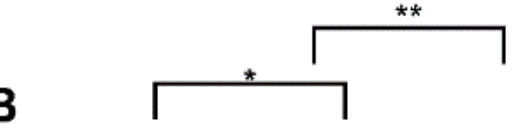

E

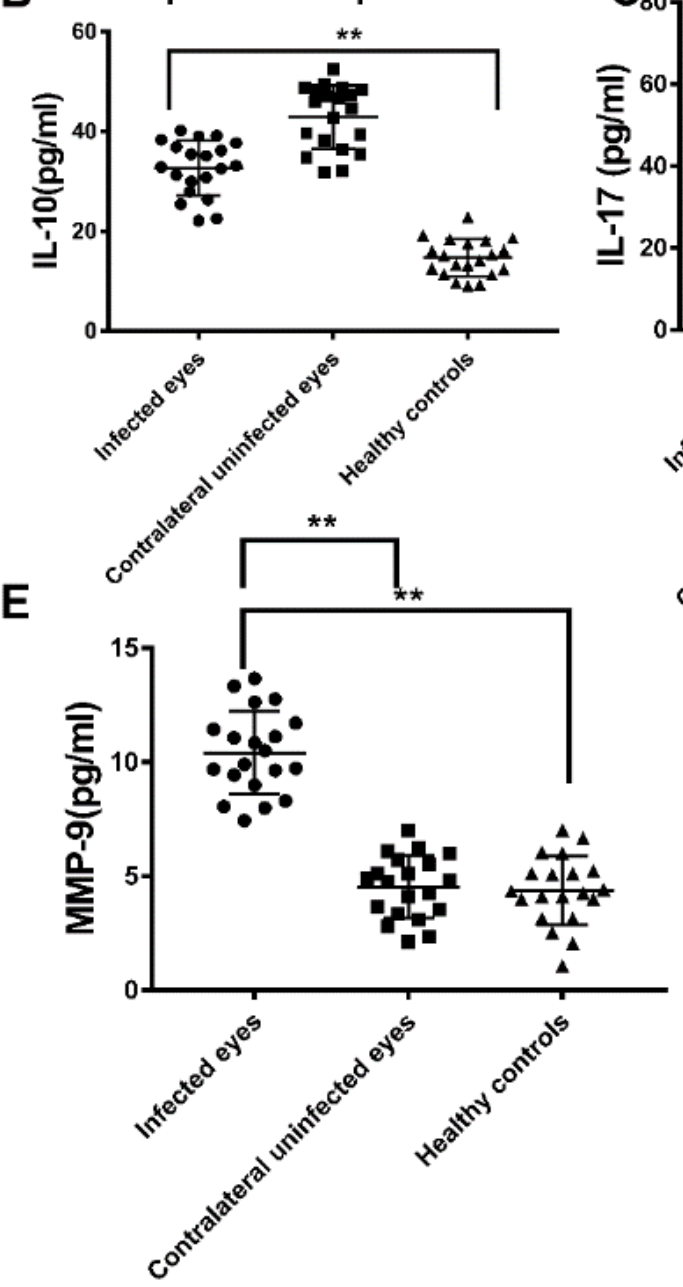

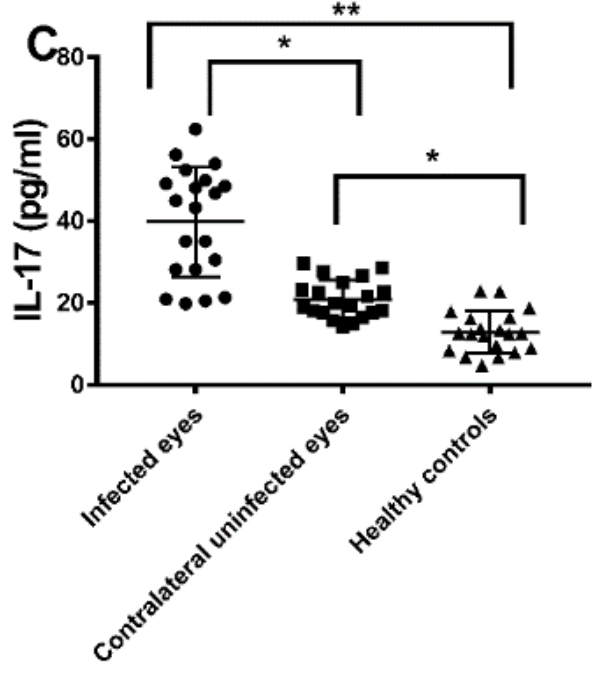

Figure 2

Inflammatory cytokines and MMP-9 levels in the tears of FK bilateral eyes and healthy control eyes Notes:*夫 represents $\mathrm{P}<0.01$, * represents $\mathrm{P}<0.05$, A P value of less than 0.05 was considered statistically significant. Abbreviations : MMP-9,Matrix metalloproteinase-9; FK ,Fungal keratitis. 


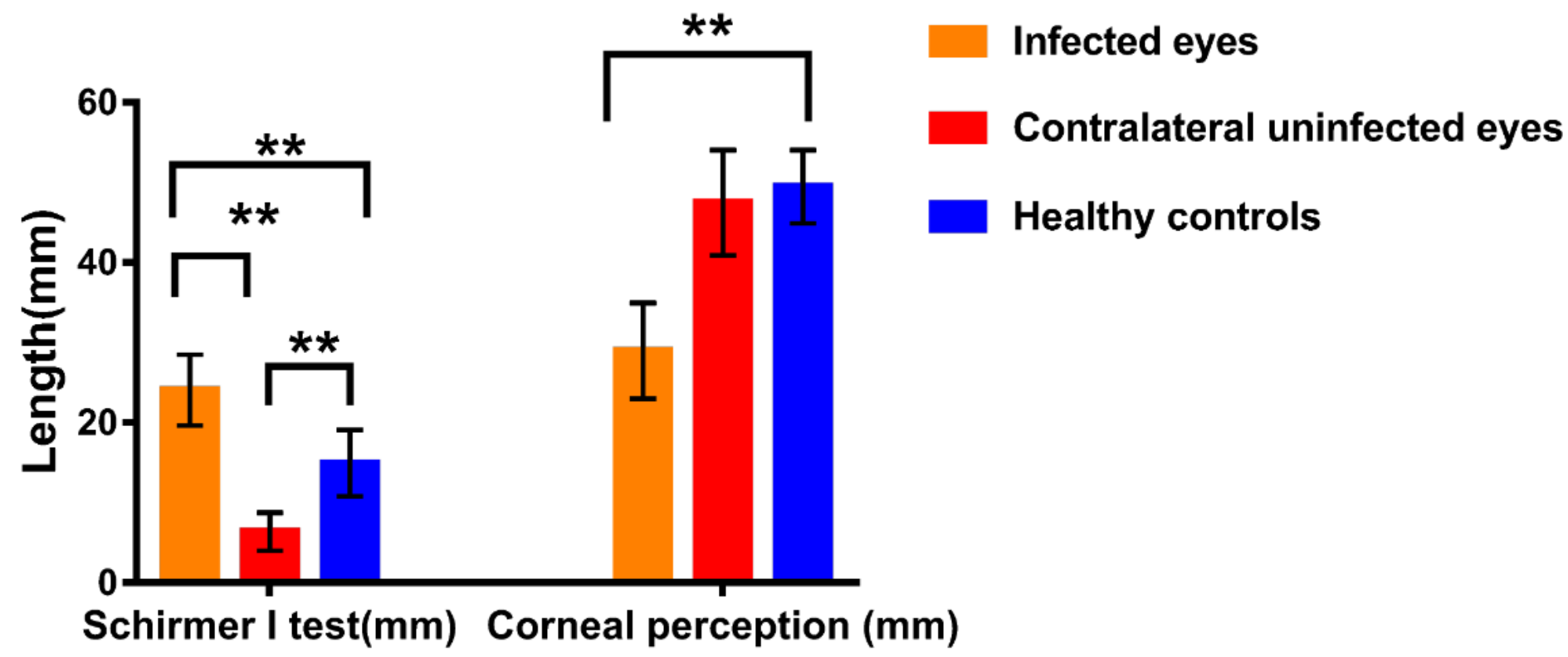

Figure 3

The comparison of Schirmer Strip, corneal perception ,tear breakup time between FK group and healthy group Notes: ** represents $\mathrm{P}<0.01$, A P value of less than 0.05 was considered statistically significant. Abbreviations :TBUT, tear film rupture time; FK ,Fungal keratitis.

A

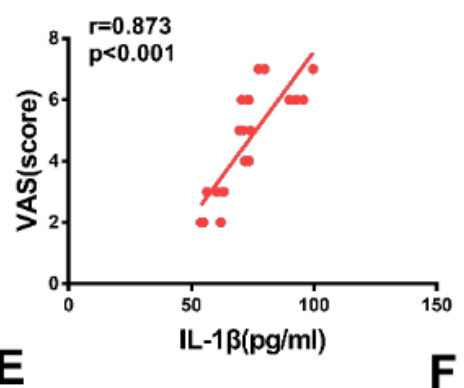

E

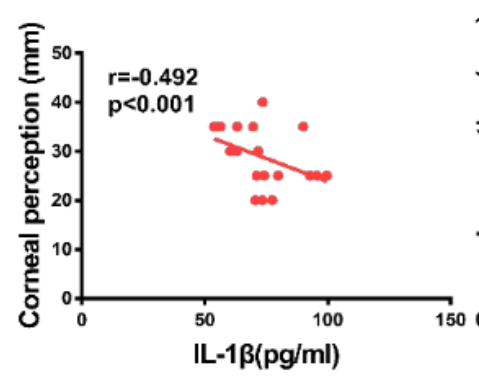

B

$\mathbf{F}$
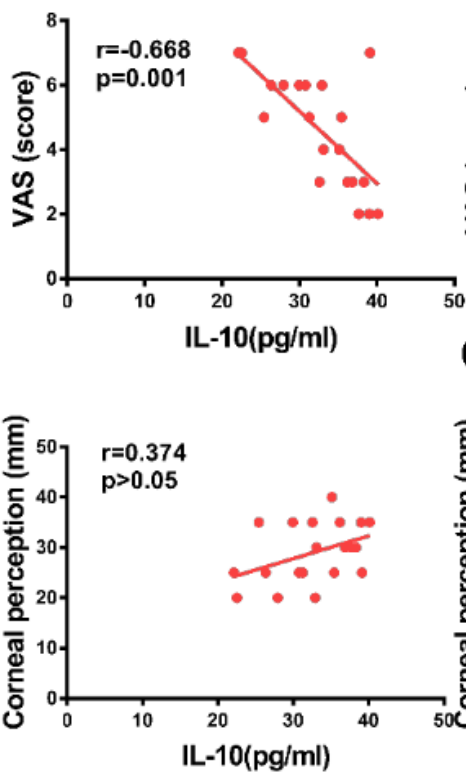

C
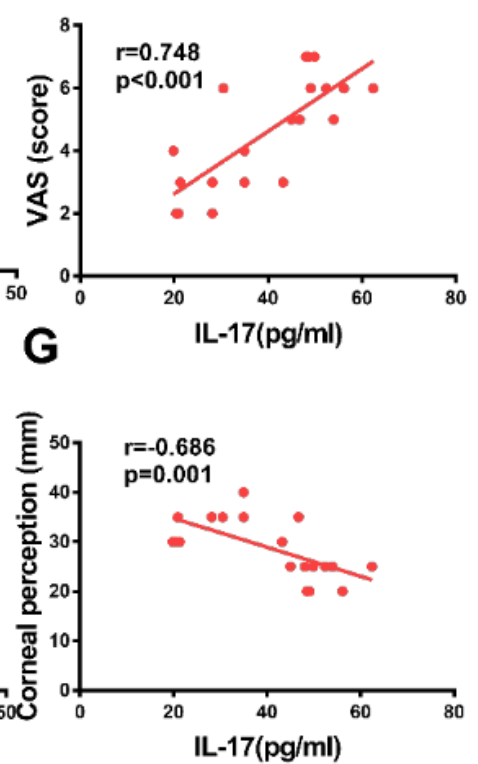

D
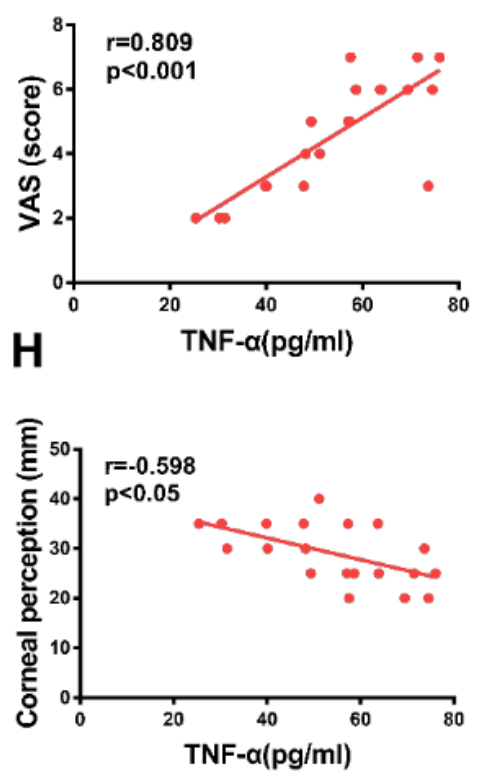

Figure 4 
Correlation of IL-1 $\beta$ (Fig.4A),IL-10(Fig.4B), IL-17(Fig.4C),TNF - $a$ (Fig.4D) with VAS scores; Correlation of IL1 $\beta$ (Fig.4E),IL-10(Fig.4F), IL-17(Fig.4G),TNF - $a$ (Fig.4H) with corneal perception. Notes: The relationship between corneal perception and VAS and inflammatory cytokines in FK-affected eyes are all analyzed by Spearman correlation. A P value of less than 0.05 was considered statistically significant. Abbreviations :IL, Interleukin; TNF, Tumor necrosis factor; VAS, Visual analogue scale.

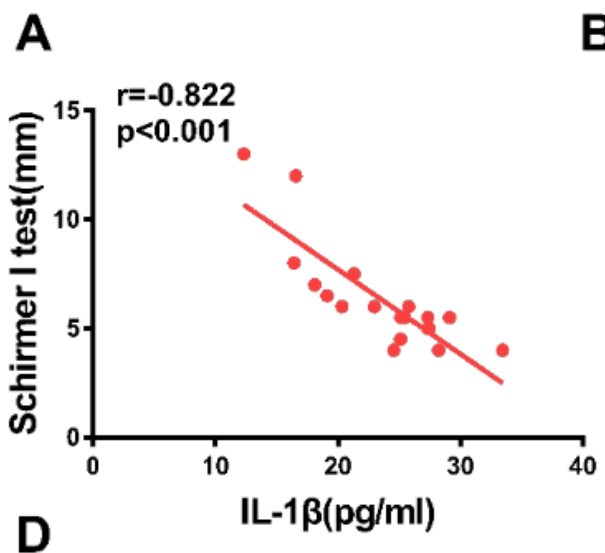

B

C
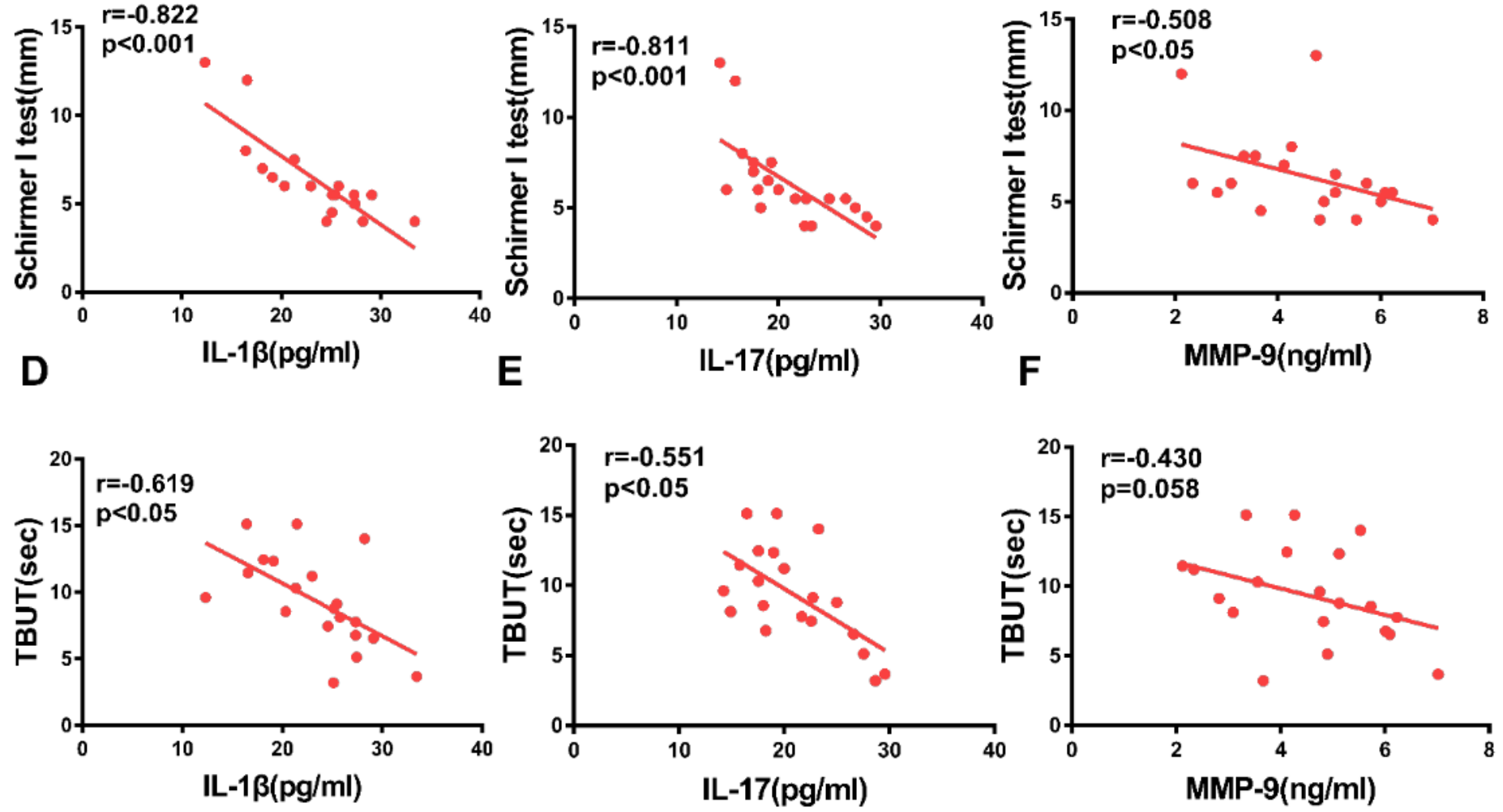

Figure 5

Correlation of IL-1 $\beta$ (Fig.5A), IL-17(Fig.5B),MMP-9 (Fig.5C) with Schirmer I test; Correlation of IL1 $\beta$ (Fig.5D), IL-17(Fig.5E),MMP-9 (Fig.5F) with TBUT. Notes: The relationship between tear film function and inflammatory cytokines on the contralateral side of FK was analyzed by Spearman correlation. A P value of less than 0.05 was considered statistically significant. Abbreviations :IL, Interleukin;MMP-9, Matrix metalloproteinase-9; TBUT, tear breakup time. 
A $\quad$ B

C
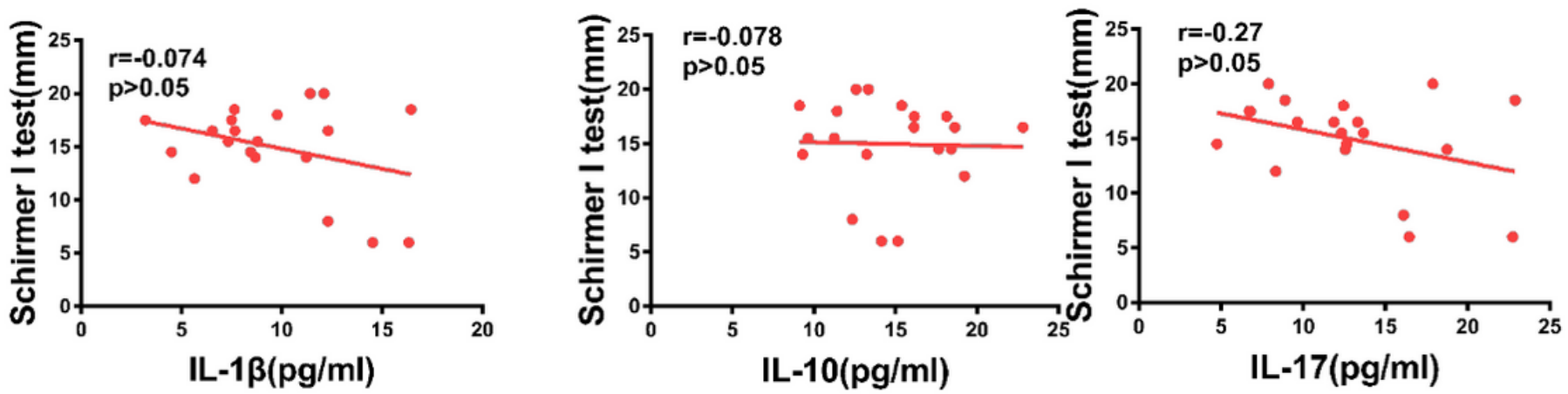

D

$\mathbf{E}$

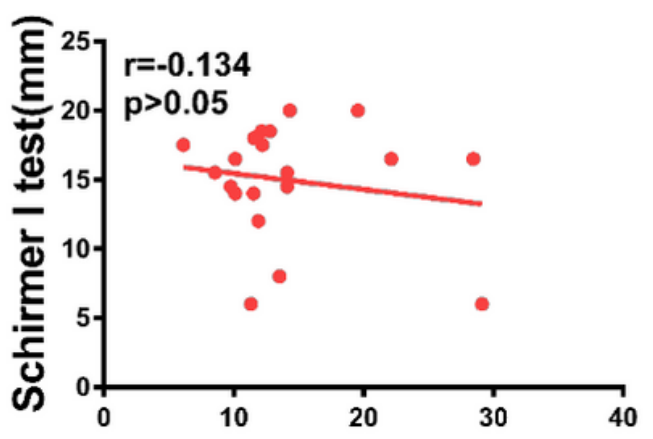

TNF- $\alpha(p g / m l)$

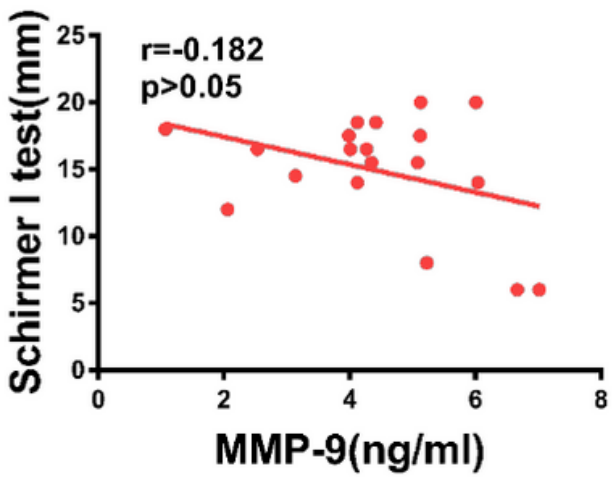

MMP-9(ng/ml)

Figure 6

Correlation of tear cytokines with Schirmer Strip in healthy controls Notes: The relationship between filter paper infiltration length and inflammatory cytokines in the healthy control group was analyzed by Spearman correlation. A P value of less than 0.05 was considered statistically significant. Abbreviations :IL, Interleukin;MMP-9, Matrix metalloproteinase-9; TNF, Tumor necrosis factor. 
A

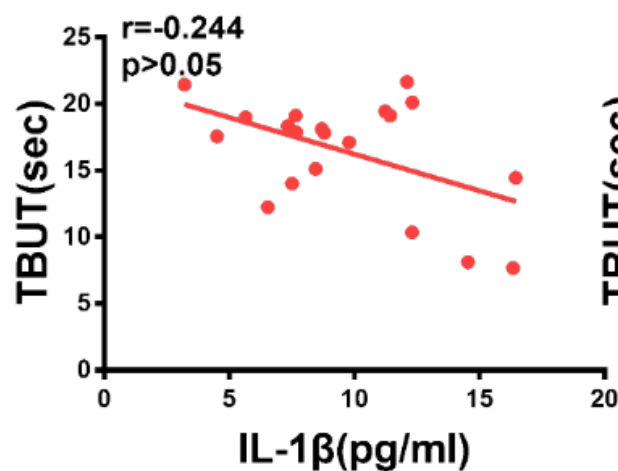

D

$\mathrm{IL}-1 \beta(\mathrm{pg} / \mathrm{ml})$

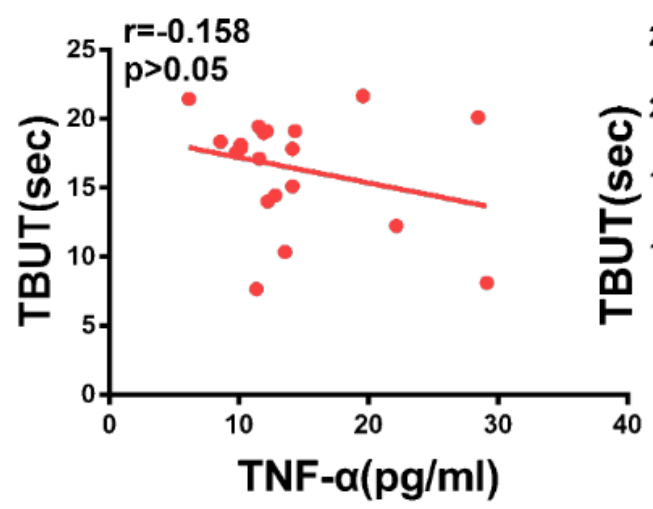

C

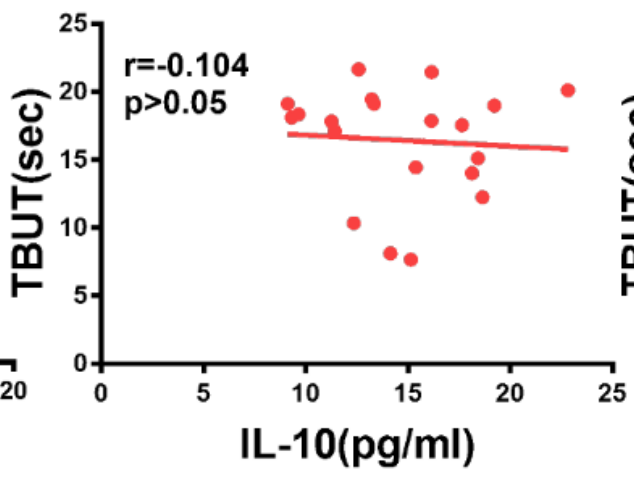

$\mathbf{E}$

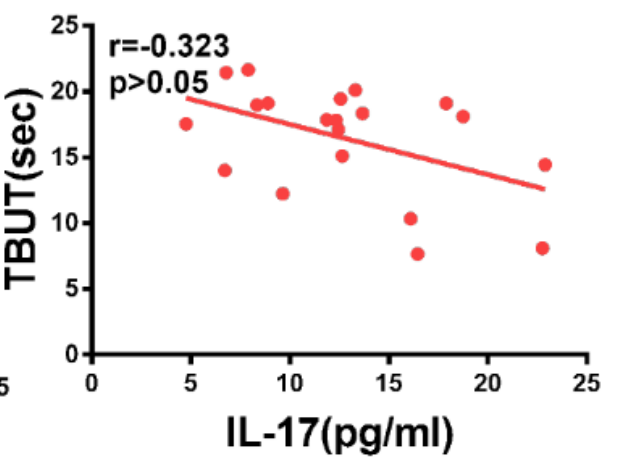

IL-17(pg/ml)

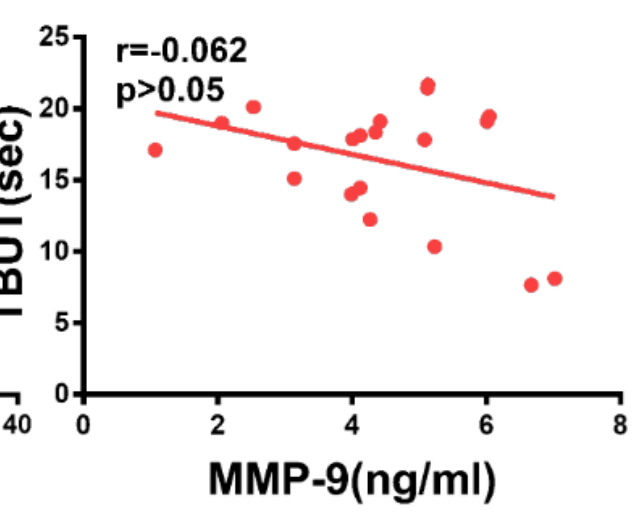

Figure 7

Correlation of tear cytokines with TBUT in healthy controls Notes: The relationship between filter paper infiltration length and inflammatory cytokines in the healthy control group was analyzed by Spearman correlation. A P value of less than 0.05 was considered statistically significant. Abbreviations : IL, Interleukin;MMP-9, Matrix metalloproteinase-9; TNF, Tumor necrosis factor; TBUT, tear breakup time. 